\title{
The paradox of productivity during quarantine: an agent-based simulation
}

\author{
Peter $\mathrm{Hardy}^{1}$, Leandro Soriano Marcolino ${ }^{1}$, and José F. Fontanari ${ }^{2, \mathrm{a}}{ }_{\mathbb{D}}$ \\ 1 School of Computing and Communications, Lancaster University, Lancaster, LA LA1 4WA, UK \\ 2 Instituto de Física de São Carlos, Universidade de São Paulo, Caixa Postal 369, São Carlos, São Paulo 13560-970, Brazil
}

Received 21 August 2020 / Accepted 18 December 2020 / Published online 29 January 2021

(C) EDP Sciences, SIF and Springer-Verlag GmbH Germany, part of Springer Nature 2021

\begin{abstract}
Economies across the globe were brought to their knees due to lockdowns and social restriction measures to contain the spread of the SARS-CoV-2, despite the quick switch to remote working. This downfall may be partially explained by the "water cooler effect", which holds that higher levels of social interaction lead to higher productivity due to a boost in people's mood. Somewhat paradoxically, however, there are reports of increased productivity in the remote working scenario. Here we address quantitatively this issue using a variety of experimental findings of social psychology that address the interplay between mood, social interaction and productivity to set forth an agent-based model for a workplace composed of extrovert and introvert agent stereotypes that differ solely on their propensities to initiate a social interaction. We find that the effects of curtailing social interactions depend on the proportion of the stereotypes in the working group: while the social restriction measures always have a negative impact on the productivity of groups composed predominantly of introverts, they may actually improve the productivity of groups composed predominantly of extroverts. Our results offer a proof of concept that the paradox of productivity during quarantine can be explained by taking into account the distinct effects of the social distancing measures on extroverts and introverts.
\end{abstract}

\section{Introduction}

The interest on the trade-off between work productivity and social interaction is not a present-day fad, as attested by this verse of the 19th century writer Maria Edgeworth: "All work and no play makes Jack a dull boy, All play and no work makes Jack a mere toy" [1]. In fact, this is a staple subject of social psychology that addresses the influence of loneliness or, more generally, of mood on human cognitive function, often with discrepant findings (see, e.g., $[2,3]$ ). Recent lockdowns and social restriction measures that isolated workers from their peers have placed these issues at the forefront of public attention, with news outlets reminding their customers that they do not need to be productive, and to expect a reduction in overall productivity during this time. But why is it that productivity would decrease during a period that one would think people have more time on their hands than ever before?

Typical answers to this question are in line with the so-called "water cooler effect" that has been highlighted (and satirized) in how effective placing an inanimate object for people to congregate around can stir up casual conversations, with many psychologists believing that this can also increase company productivity [4]. However, this is not an obvious conclusion since for a singular worker the extra down time communicating

\footnotetext{
a e-mail: fontanari@ifsc.usp.br (corresponding author)
}

may actually detract from its productivity as a whole. The economic literature offers more solid explanations for the positive correlation between social interaction or communication among co-workers and productivity [5]. For instance, the peer pressure that generates feelings of guilt or shame when one's performance falls short of the expectation may increase focus on the work and hence increase productivity $[6,7]$. In addition, it is through social interactions that coworkers learn from each other and build up skills that they otherwise would not have. This so-called knowledge spillover is the usual explanation for the strong link between economic growth and the concentration of people in cities $[8,9]$.

Here we address quantitatively the productivity and social interaction issue using an agent-based model to simulate a workplace scenario where the agents exhibit two social stereotypes, viz., extroverts and introverts, that differ solely on their propensities to initiate a conversation. This is in accord with the well-established view that extroverts and introverts equally enjoy engaging in social interactions and in other pleasurable activities, but differ in the likelihood that they would be the instigators of those activities [10]. Social distancing is modeled by controlling the number of attempts an agent makes to find a conversation partner. The motivation to work (mood) is assumed to increase with the time spent talking and decrease with the time spent alone. Moreover, the instantaneous productivity of a lone agent increases with its motivation to work. These 
assumptions are grounded on the unsurprising findings that positive affect (i.e., the propensity to be in a good mood) increases significantly after social interaction [11,12], facilitates creative problem solving [13] and boosts productivity in general $[14,15]$.

We find that the effect of curtailing social interactions depends on the proportion of the different stereotypes in the group. For instance, while social restriction measures always have a negative impact on the productivity of groups composed predominantly of introverts, they may actually improve the productivity of groups composed predominantly of extroverts. In addition, within a same working group the productivity of the two stereotypes is affected differently by those measures. These results may explain the paradoxical findings that productivity is increased in some remote work experiments [16].

\section{Model}

We model the dynamics of a group of $N$ agents that interact socially and work during a 480-min (8-h) workday. We first make the fair assumption that for all agents they are more productive when they are motivated (i.e., in a good mood to work) [13-15], and their motivations increase when they participated in some form of social interaction [11,12]. In this framework, motivation has both an emotional dimension, in the sense that talking with peers improves the mood of the agent and helps it to focus on the task, and an informational dimension, in that talking with peers may result in the acquisition of valuable information that can help the agent to consummate its task, which is the knowledge spillover mechanism mentioned previously.

We assume that the motivation of agent $k=$ $1, \ldots, N$ is determined by the integer parameter $L_{k}=$ $1,2, \ldots, L_{\max }$ and that at the beginning of the day, the agents' motivations are the lowest, i.e., $L_{k}=1$. In addition, we assume that the instantaneous productivity of an agent at time $t=0,1, \ldots, T$ is $P_{k}(t)=L_{k}-1$ if agent $k$ is not talking and $P_{k}(t)=0$ if agent $k$ is talking. This is in line with the experimental findings that being with other people would be associated with poorer cognitive task performance than when being alone [3]. Since we measure time in minutes, we set $T=480$. Hence, at time $t=0$ the instantaneous productivity is zero for all agents.

The only way an agent can increase its motivation and, consequently, its productivity, is by engaging in social interaction. We introduce two different social stereotypes our agents can be, viz., extroverts and introverts, which determine their propensities to seek and engage in social interaction. In particular, if agent $k$ has stereotype $l$, where $l=e$ for extroverts and $l=i$ for introverts, the probability that it instigates a conversation is

$$
p_{k}^{l}=\left(T / \tau^{l}-L_{k}\right) /\left(T / \tau^{l}-1\right)
$$

for $L_{k} \leq T / \tau^{l}$ and $p_{k}^{l}=0$, otherwise. Here $\tau^{e}$ and $\tau^{i}$ are parameters measured in minutes that are necessary to make Eq. (1) dimensionally correct. Throughout this paper we set $\tau^{e}=1$ without loss of generality, but set $\tau^{i}>1$ in order to guarantee that $p_{k}^{e} \geq p_{k}^{i}$, i.e., that the extroverts are more likely to engage in social interaction than the introverts, in agreement with the personality theory findings [10]. In addition, $p_{k}^{e}=p_{k}^{i}=$ 1 for $L_{k}=1$ so that when the motivation of agent $k$ is at the bottom line, it will try to engage in social interaction with certainty. The propensity to instigate a conversation decreases linearly with the motivation to work until it vanishes altogether when the motivation parameter reaches a threshold value that differs for the two social stereotypes. This decrease is justified because no work is done while the agent is talking and so we should expect that the higher the agent's motivation to work is, the lower its propensity to socialize. The different threshold values are necessary to account for the existence of two unambiguous stereotypes.

Regardless of its stereotype, once a target agent decides to instigate a conversation, it selects a number $m$ of contact attempts, where $m=0,1, \ldots$ is a random variable drawn from a Poisson distribution of parameter $q$. In each contact attempt, a peer is selected at random among the $N-1$ agents in the group and, in case the selected agent is not in a conversation at that time, a conversation is initiated and the target agent halts its search for a partner. The duration of the conversation $d$ is given by a random integer selected uniformly in $\{1, \ldots, D\}$. We note that a conversation involves two agents only and the agent that is approached by the target agent is obliged to accept the interaction, regardless of its motivation and stereotype. This pro-social behavior is chosen in order to not further complicate the model, but it can be justified in terms of workplace social norms [17].

To complete the model we need to give a prescription for changing the motivation parameter $L_{k}$ with $k=1, \ldots, N$, which is the central factor in the determination of the social behavior of the agents. We assume simply that the motivation of agent $k$ increases by one unit for each minute it spends in a conversation and decreases by one unit for each minute it spends working alone. This is in accordance with the previously mentioned experimental findings that mood improves after social interaction $[11,12]$ and that being alone rather than with others while doing a task results in worse mood but better cognitive task performance [3]. Moreover, we set $L_{k}=1$ as the lower bound of the motivation parameter so that a lone agent $k$ at the motivation bottom line $L_{k}=1$ cannot have its motivation reduced any further.

We note that our prescription to change the motivation of the agents implies that the motivation parameter is bounded from above by $L_{\max }=480$, so that the probability that an extroverted agent instigates a conversation, Eq. (1), is never zero during the eighthour workday. This produces a qualitative distinction between the two stereotypes since the extroverts are, in principle, always willing to initiate a social interaction 
(i.e., $p_{k}^{e}>0$ ), whereas the introverts will not instigate a conversation if their motivation to work is above the threshold $T / \tau^{i}$. Throughout this paper we set $\tau^{i}=5$ but note that this choice is immaterial, provided that $T / \tau^{i}$ is sufficiently distinct from the upper bound $T / \tau^{e}$ to justify the existence of two stereotypes.

In summary, we implement the synchronous or parallel update of the $N$ agents as follows. At $t=0$ we set $L_{k}=1$ and $P_{k}=0$ for all agents $k=1, \ldots, N$. The update procedure at time $t$ begins with the selection of a random order of update of the $N$ agents, so that at the end of the procedure all agents are updated and we can increment the time from $t$ to $t+1$. Then we check the status of the agent to be updated - the target agent - to determine if it is participating in a conversation or not. In case it is, we increment its motivation parameter by one unit. In case it is not, we decrement its motivation parameter by one unit and test whether it initiates a conversation or not using the probabilities given in Eq. (1) in accord with the stereotype of the target agent. The simulation ends at $t=T$.

\section{Results}

We consider working groups composed of $N^{e}$ extroverts and $N^{i}=N-N^{e}$ introverts and focus mainly on the mean cumulative productivity of the stereotypes at time $t=1, \ldots, T$,

$$
\Pi^{l}(t)=\frac{1}{N^{l}} \sum_{k \in \mathcal{S}^{l}} \pi_{k}(t)
$$

where

$$
\pi_{k}(t)=\frac{1}{t} \sum_{t^{\prime}=0}^{t} P_{k}\left(t^{\prime}\right)
$$

and the superscript $l=e, i$ specifies the social stereotypes of the agents, as before. Here the sum over $k$ is restricted to the subset $\mathcal{S}^{l}$ of agents with stereotype $l$, whose cardinality is $N^{l}$. We recall that $P_{k}(0)=0$ for all $k$. The mean cumulative productivity of the whole group is simply $\Pi^{w}=\left(N^{e} \Pi^{e}+N^{i} \Pi^{i}\right) / N$, where we have omitted the dependence on $t$ for simplicity. It is also of interest to know the mean motivation of the stereotype subgroups,

$$
\Lambda^{l}(t)=\frac{1}{N^{l}} \sum_{k \in \mathcal{S}^{l}} L_{k}(t)
$$

with $l=e, i$ as before. Then the mean motivation of the whole group is $\Lambda^{w}=\left(N^{e} \Lambda^{e}+N^{i} \Lambda^{i}\right) / N$.

Our main interest here is $\Pi^{l}(t)$ and $\Lambda^{l}(t)$ evaluated at the end of the day, i.e, at $t=T$. These quantities are averaged over $10^{3}$ independent runs for each setting of the model parameters. In particular, in the following we vary the fraction of extroverts $\eta=N^{e} / N$ and the mean number of contact attempts $q$, but fix the group size to
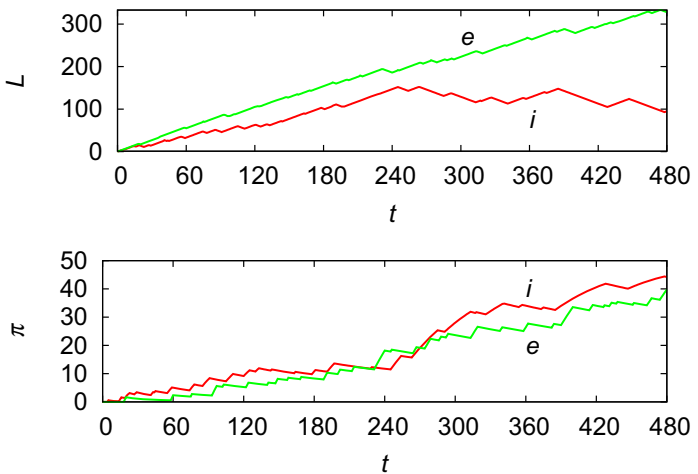

Fig. 1 Motivation (upper panel) and mean cumulative productivity (lower panel) of typical extrovert (e) and introvert $(i)$ agents for a group of size $N=100$ with equal ratio of extroverts and introverts, i.e., $\eta=0.5$. The maximum duration of a conversation is $D=20$ and the mean number of contact attempts is $q=2$

$N=100$ and the maximum duration of a conversation to $D=20$. As already pointed out, we set $\tau^{e}=1$ and $\tau^{i}=5$.

To better appreciate the assumptions of the model, Fig. 1 shows typical time dependences of the motivation and mean cumulative productivity of two agents with distinct stereotypes for a single run. The time intervals where the mean cumulative productivity decreases correspond to the periods when the agent is participating in a social interaction and are associated with the increase of its motivation. We note that, whereas the cumulative productivity is a non-decreasing function of $t$, the mean cumulative productivity, Eq. (3), decreases with increasing $t$ in the time intervals where the instantaneous productivity of the agent is zero. In addition, the motivation decreases in the periods where the productivity increases. Most of the time in this run, the introvert agent exhibits a higher mean productivity than the extrovert agent, despite its lower motivation. In fact, the motivation parameter of the introvert stabilizes and fluctuates around the value $L=T / \tau^{i}=$ 96, whereas the motivation parameter of the extrovert shows a tendency to increase linearly with $t$ on average. It is easy to understand the behavior pattern of the introvert's motivation if we note that an introvert agent with $L \geq T / \tau^{i}$ will not attempt to instigate any conversation and so the tendency of its motivation is to decrease only, except for the events when another agent engages it in a conversation.

Figure 2 shows the influence of the fraction of extroverts $\eta$ on the motivation and mean cumulative productivity of the agents at the end of the day. This figure reveals some interesting features. First and foremost, the insertion of extroverts in groups composed predominantly of introverts results in a significant increase of the mean productivity of the introverts, whereas the mean productivity of the extroverts is barely changed. Too many extroverts, however, cause a decrease on the productivity of both stereotypes, although they considerably boost the motivation of the introverts. In addi- 

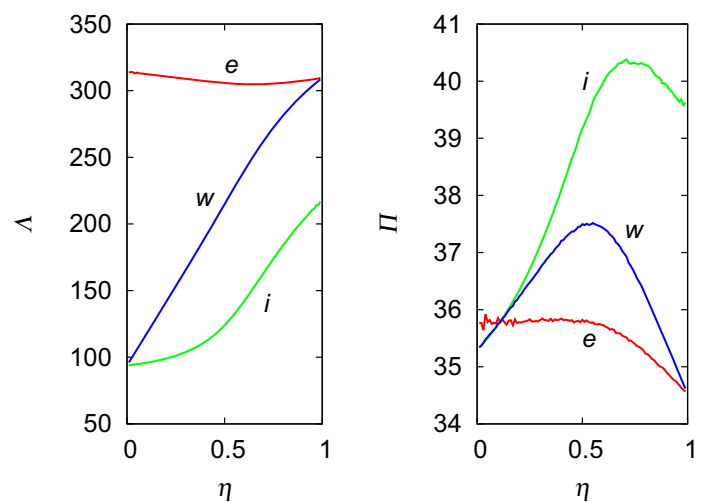

Fig. 2 Effect of the fraction of extroverts on the mean motivation (left panel) and on the mean cumulative productivity (right panel) at the end of the day of the subgroups of extroverts $(e)$ and introverts $(i)$, and of the whole group $(w)$. The total number of agents is $N=100$, the maximum duration of a conversation is $D=20$ and the mean number of contact attempts is $q=2$

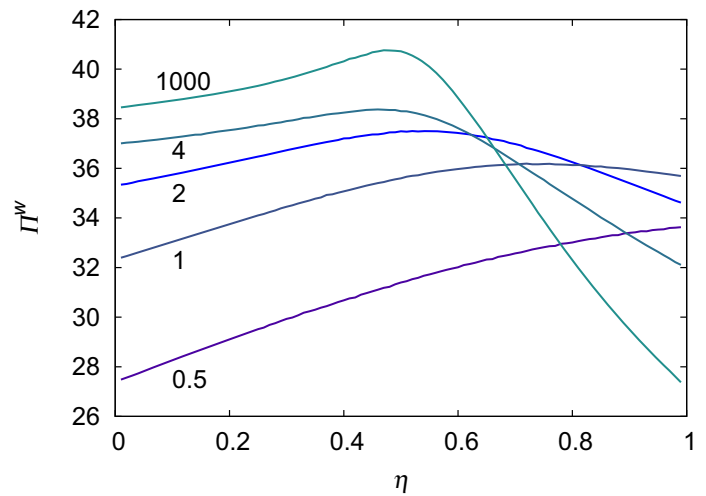

Fig. 3 Mean cumulative productivity of the whole group at the end of the day as function of the fraction of extroverts for mean number of contact attempts $q=0.5,1,2,4$ and 1000 , as indicated. The total number of agents is $N=100$ and the maximum duration of a conversation is $D=20$

tion, there is an optimum group composition $(\eta \approx 0.5$ for the parameters used in Fig. 2) that maximizes the total productivity of the group.

In Fig. 3, we examine the influence of the mean number of contact attempts $q$ on the optimum composition of the group. For instance, for small $q$, say $q=0.5$, it is difficult to find conversation partners to increase the agents' motivations and so the highest total productivity is achieved by all-extroverts groups, since this stereotype seeks for partners more frequently than the introverts. As $q$ increases, the odds of finding a conversation partner increases as well, and so it becomes advantageous to insert a few introverts in the group. For very large $q$, so that the target agent attempts to contact all the other agents in the group, the maximum total productivity occurs for $\eta \approx 0.47$. For groups composed predominantly of introverts $(\eta<0.5)$, increase of $q$ and $\eta$ lead always to higher total productivity. However, the scenario is more complicated for groups com-

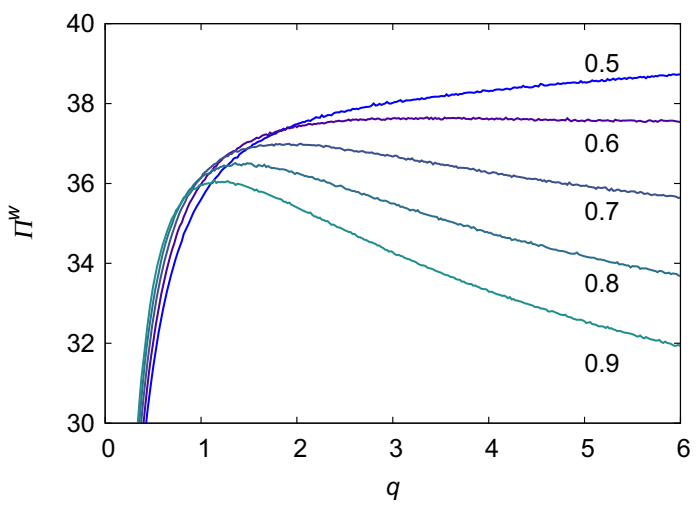

Fig. 4 Effect of the mean number of attempts to establish social interaction on the mean cumulative productivity of the whole group at the end of the day for the fraction of extroverts $\eta=0.5,0.6,0.7,0.8$ and 0.9 , as indicated. The total number of agents is $N=100$ and the maximum duration of a conversation is $D=20$

posed predominantly of extroverts. In fact, for $\eta>0.5$ our results indicate that there is an optimum value of the mean number of contact attempts that maximizes the total productivity. For instance, this optimum value is located between $q=1$ and $q=2$ for $\eta \approx 1$.

It is worthwhile to examine the effects of the parameter $q$ in more detail. In the context of the 2020 coronavirus pandemic, this is the leading parameter of the model since, at least for those adhering to social distancing norms, the mean number of attempts to interact socially was considerably curtailed during the pandemic. Accordingly, Fig. 4 shows that a moderate decrease of $q$ actually increases the productivity of the group for $\eta>0.5$. For instance, for $\eta>0.8$ the decrease from $q=6$ to $q=1$ results in a productivity increase of about $10 \%$, which is in agreement with the experiments of Ref. [16]. We note that in both our simulations and those experiments the boost in productivity is modest, though altogether unexpected.

It is interesting to look at how the productivities of the two stereotypes within a same working group are affected by the social restrictions. Figure 5 shows that, for $\eta=0.5$, a moderate decrease of $q$ reduces the motivation of both stereotypes as well as the mean productivity of the introverts, but increases the mean productivity of the extroverts. This finding may explain sporadic personal reports of heightening productivity in the quarantine. Further decrease of $q$ leading to a scenario where social interactions happen very rarely results in a sharp drop of the mean productivity of both stereotypes. These results add to the message that mathematical and computational models of the effects of the 2020 coronavirus pandemics and its palliative measures (e.g., social distancing) need to take into account the distinct age and socioeconomic segments of the population [18]. In fact, we argue here that the individuals' psychological traits play an important role regarding work productivity and hence ought to 

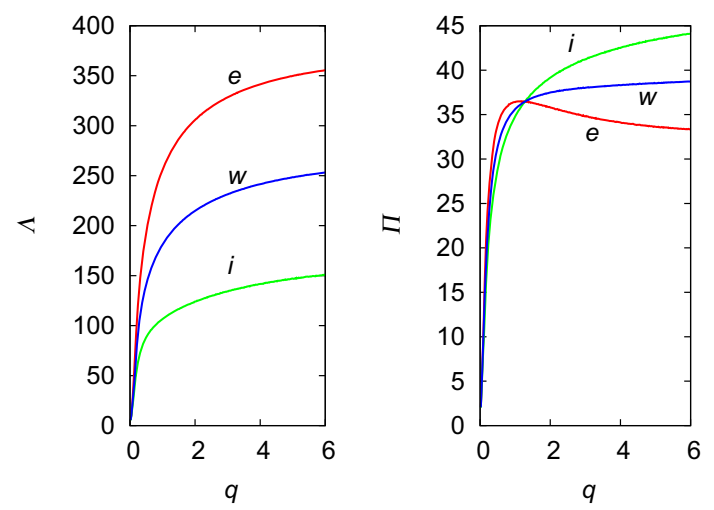

Fig. 5 Effect of the mean number of attempts to establish social interaction on the mean motivation (left panel) and on the mean cumulative productivity (right panel) at the end of the day of the subgroups of extroverts $(e)$ and introverts $(i)$, and of the whole group $(w)$. The total number of agents is $N=100$, the maximum duration of a conversation is $D=20$ and the fraction of extroverts is $\eta=0.5$

be considered on an assessment of the socio-economic implications of the pandemics.

A word is in order about the effects of the parameters $N$ and $D$ that determine the group size and the maximum duration of a conversation, respectively. Reducing the group size has no effect on our results and our choice of fairly large groups $(N=100)$ is so as to smooth out the variation of the fraction of extroverts $\eta$. However, increase of the parameter $D$ has a strong effect on the productivity of the extroverts. For instance, for long conversations, say $D=60$, all-introverts groups yield the highest total productivity regardless of the number of contact attempts. Our choice $D=20$, which corresponds to conversations of average duration $\bar{d}=10$, are meant to model a "water cooler talk" scenario.

\section{Conclusion}

In the spirit of sociophysics [19,20] and computational social science [21], our agent-based model builds on a variety of experimental findings of social psychology on the interplay between mood, social interaction and productivity $[3,11-15]$ to advance an explanation for the mostly anecdotal reports of increased productivity in the present-day pandemics scenario as well as for the more solid findings that productivity is increased in some remote work experiments [16]. These are puzzling findings because they challenge the established view that communication among co-workers in the workplace increases productivity either through peer pressure or knowledge spillover [5-8]. The key ingredient to explain this puzzle is the observation that the population is heterogeneous with respect to the individuals' propensities to engage in social interactions [10] and that introverts and extroverts are affected differently by the social distancing and quarantine policies.
Since the social psychology studies that support our model assumptions are correlational, they do not offer specific quantitative relationships between mood and productivity or between mood and propensity to initiate a conversation that are necessary to implement a simulation of the workplace scenario. Hence, to minimize spurious effects due to the, to some extent, arbitrary choices of the functional relationships between those variables, our model assumes linear relationships only. However, provided different choices accord with the correlational studies, we expect our conclusions to remain valid. We stress that, rather than offering a precise quantitative characterization of the workplace dynamics, our model offers a proof of concept that quarantining and social distancing may boost the productivity of extroverted people, thus throwing light on the paradox of productivity during quarantine.

Acknowledgements The research of JFF was supported in part by Grant no. 2020/03041-3, Fundação de Amparo à Pesquisa do Estado de São Paulo (FAPESP) and by Grant no. 305058/2017-7, Conselho Nacional de Desenvolvimento Científico e Tecnológico (CNPq).

\section{Author contributions}

All the authors contributed equally to the paper.

Data Availability Statement This manuscript has no associated data or the data will not be deposited. [Authors' comment: The results and data presented in the manuscript can be replicated using the numerical procedures described in the text.]

\section{References}

1. M. Edgeworth, Harry and Lucy Concluded: Being the Last Part of Early Lessons (Munroe \& Francis, Boston, 1825)

2. J.T. Cacioppo, L.C. Hawkley, Trends Cogn. Sci. 13, 447-454 (2009)

3. S. von Stumm, Cogn. Emot. 32, 1545-1558 (2018)

4. A. Pentland, Harv. Bus. Rev. 87, 37 (2009)

5. T. Cornelissen, C. Dustmann, U. Schönberg, Am. Econ. Rev. 107, 425-456 (2017)

6. E. Kandel, E.P. Lazear, J. Polit. Econ. 100, 801-817 (1992)

7. O. Bandiera, I. Barankay, I. Rasul, Rev. Econ. Stud. 77, 417-458 (2010)

8. M.O. Feldman, D.B. Audretsch, Eur. Econ. Rev. 43, 409-429 (1999)

9. L.M.A. Bettencourt, J. Lobo, D. Helbing, C. Kühnert, G.B. West, Proc. Natl. Acad. Sci. USA 104, 7301-7306 (2007)

10. J. Feist, G. Feist, R. Tomi-Ann, Theories of Personality (McGraw-Hill, New York, 2012)

11. D.L. Phillips, Am. J. Sociol. 72, 479-488 (1967)

12. C.W. McIntyre, D. Watson, L.A. Clark, S.A. Cross, Bull. Psychon. Soc. 29, 67-70 (1991) 
13. A.M. Isen, K.A. Daubman, G.P. Nowicki, J. Pers. Soc. Psychol. 52, 1122-1131 (1987)

14. H.C. Ellis, P.W. Ashbrook, Affect, Cognition, and Social Behavior, Edited by K. Fiedler, J. Forgas (Hogrefe, Göttingen, 1988), pp. 35-42

15. A. Oswald, E. Proto, D. Sgroi, J. Labor. Econ. 33, 789 $822(2015)$

16. N. Bloom, J. Liang, J. Roberts, Z.J. Ying, Q. J. Econ. 130, 165-218 (2015)

17. G. Johns, A.M. Saks, Organizational Behaviour: Understanding and Managing Life at Work (Pearson/Prentice Hall, Toronto, 2005)

18. N. Bellomo, R. Bingham, M.A.J. Chaplain, G. Dosi, G. Forni, D.A. Knopoff, J. Lowengrub, R. Twarock, M.E. Virgillito, Math. Models Methods Appl. Sci. 30, 15911651 (2020)
19. M. Perc, J.J. Jordan, D.G. Rand, Z. Wang, S. Boccaletti, A. Szolnoki, Phys. Rep. 687, 1-51 (2017)

20. M. Perc, Sci. Rep. 9, 16549 (2019)

21. D. Lazer, A. Pentland, L. Adamic, S. Aral, A.L. Barabasi, D. Brewer, N. Christakis, N. Contractor, J. Fowler, M. Gutmann, T. Jebara, G. King, M. Macy, D. Roy, M. Van Alstyne, Science 323, 721-723 (2009) 